\section{Oncology: A Multidisciplinary Textbook}

Edited by Alan Horwich. London: Chapman \& Hall, 1994, 703 pp. £65.00. ISBN 0-412-552507.

In most libraries and book shops, the interested reader now has a number of multiauthor comprehensive textbooks of oncology from which to choose. What is different about this one and why should anyone choose it?

Firstly, this book is unusual in that it is largely derived from a single institute, The Royal Marsden Hospital and Institute of Cancer Research. Secondly, it purports to be multidisciplinary, i.e. representing input from the surgeons and clinical and medical oncologists who make up the individual tumor units which have made major contributions to the Institute's oncology course. As Professor Horwich explains, the book 'represents the didactic components of this course'. It is intended as an introduction to the subject for those embarking on a career in oncology, and a source of information for those in allied specialities. How successful is it in achieving its aims?

Like the curate's egg, it is good in parts. The first third of the book describes 'the principles of oncology'. The first three chapters cover epidemiology and molecular and cell biology of cancer, and then the remaining chapters in this first section are orientated towards clinical issues, including diagnosis, treatment and supportive care. While some of these can obviously be found in other books, the standard in the section is generally high, and some of the chapters are particularly creditworthy. Chapters such as New imaging techniques in radiology (Dr R Guy), The pyschological toll of cancer (Dr S Greer) and The scope of cancer nursing (Dr J Corner) do give this book a distinctive flavour; there is also an excellent section called The Principles of cytokine therapy (Drs Taylor and Gore).

The second section of about 450 pages comprises 'a systemic reveiw of oncology'. It contains 29 chapters dealing with different tumour types. Here some chapters have succeeded more than others. Perhaps this reflects the fact that the chapters vary in the extent to which clinicians from different specialities have contributed. Some chapters do carry a bias towards the major interest of the leading author, be he a surgical or a non-surgical oncologist. For example, as regards the use of chemotherapy the reader can find great detail on this in the chapter on gastric cancer, whereas in the section on adult sarcoma the topic is given short shrift. Nevertheless each of these chapters does provide a wealth of information, particularly on the pathology, diagnosis and clinical features of each disease type. The chapters vary in their methods of presentation, and one feature which is missing from the majority is a simple table of treatment recommendations which might be of benefit to the casual reader.

On the whole, however, this is a book which could be of benefit to a wide range of individuals. It does provide an upto-date review of current treatment trends and it represents a wealth of experience and expertise from the large number of authors. The concern of course with any book of this size is that it quickly becomes dated. Perhaps the task of the combined authorship in the future will be to provide a balanced update of progress at regular intervals.

\section{Professor SB Kaye}

Cytokines in Hemopoiesis, Oncology, and Immunology III Edited by M. Freund, H. Link, R. Schmidt and K. Welte. Berlin: Springer, 1994. ISBN 0-387-57733X.

This useful book is a mine of information, especially for those interested in clinical studies of haemopoietic growth factors. It has a wide coverage of many aspects of cytokine research in laboratory and clinic, with 711 pages and over 80 chapters divided into 12 sections. An excellent review on the role of growth factors in leukaemia development by Donald Metcalf provides an introduction. The first section then describes 'new cytokines', such as stem cell factor and IL-11 and IL-12, and early clinical trials with cytokines such as IL3 , IL-4 and IL-6. Like the majority of books in the fastmoving cytokine field, particularly ones with so many authors, the contents have a tendency to be out of date. This is particularly noticeable in this section. It would, for instance, have been useful to review the promising preclinical studies of IL-12 in cancer models and cytokine gene therapy. The next section, on the use of haemopoietic growth factors to overcome the cytopenic effects of chemotherapy, describes in detail important randomised trials of recombinant G-CSF, and some studies on erythropoietin and GM-CSF. This section concludes with a cost-benefit analysis of G-CSF in patients receiving chemotherapy for small-cell lung cancer (the cost savings of adding G-CSF to the treatment protocol of small-cell lung cancer being estimated at $13 \%$ ).

Next comes a series of papers concerning peripheral blood progenitor cell support and an interesting and comprehensive section on haemopoietic growth factors in acute myeloid leukaemia and myelodysplastic syndrome. The overall theme of haemopoietic factors is continued with sections on idiopathic and congenital neutropenias (with case histories that add weight to the theory that cytokine therapy is most effective in extremely rare diseases!), and hematopoietic growth factors in infection. After a miscellaneous selection of chapters describing a multitude of effects of cytokines on normal and tumour cells in vitro, the presence of cytokines in the blood of cancer patients is detailed. Leaving the world of oncology, there is a collection of chapters covering cytokines and inflammation, with in vitro, in vivo and clinical studies described.

The coverage of cytokine therapy of cancer concentrates on results with interferon-alphas, with or without chemotherapy. There is also one chapter in this section on limb salvage by isolated perfusion of high-dose tumour necrosis factor, interferon- $\gamma$ and melphalan, showing selective destruction of tumour vasculature (effects of recombinant cytokine therapy of solid tumours are, for once, reminiscent of those obtained by Coley's mixed toxins a century ago). The book concludes with immunoregulation and immunotherapy, particularly focusing on IL-2.

This book provides an excellent update for scientists and clinicians in the cytokine field, especially those interested in therapy with haemopoietic growth factors. By covering a number of different applications of cytokine therapy, this book has a unique scope. But in spite of this comprehensive coverage, I do not think this book would be so useful to people unfamiliar to the world of cytokines and their clinical use. Each section would benefit from a short 'state-of-the-art' review, setting the individual chapters in context. This is only provided in some instances.

Dr F.R. Balkwill

The Keratinocyte Handbook

IM Leigh, EB Lane and F Watt, Cambridge University Press, Cambridge, 1995, 566pp. (hardback), ISBN 0521434165. Keratinocyte Methods

IM Leigh and F Watt, Cambridge University Press, Cambridge, 1995, 207 pp. 98paperback), ISBN 0521434165. $£ 130$ for both.

The Keratinocyte Handbook is a well-balanced review of the biology of the development and cellular differentiation in a 
variety of keratinocyte culture systems from the accepted basic technology of the $3 \mathrm{~T} 3$ feeder cell system, pioneered by Howard Green, to complex organotypic interactive models that seek to model skin (both in Part II). Part I is devoted to one chapter on ultrastructure by Karen Holbrook. This is well illustrated and gives a good morphological background A companion chapter on expression of antigenic markers might have been useful in this Part, although this is well covered in Part IV by Susan Morley and Birgitte Lane. The coverage of the biology of adhesion (Fiona Watt and others) proliferation (Robin Dover), differentiation (Ian Mackenzie, Robert Rice, Marcia Simon, Susan Morley, Beverely Dale and others) and transformation (Ximena Montano, Petra Boukamp and others) is good, and in particular, considerable emphasis is placed on interactive cell systems (Ian Mackenzie, Norbert Fusenig and Michele De Luca), which, at the present state of knowledge, are probably still essential for full phenotype expression in vitro. Markers of the differentiated phenotype are explained in Part IV and mechanisms of transformation discussed in Part V. Part VI deals with clinical applications including grafting and possibilities for gene therapy, but it would have been interesting to have seen an objective review of the practical potential of in vitro skin models in the assay of cytotoxicity, allergy, and inflammation. The production of cytokines, their cellular interactions and their role in inflammation is dealt with by Tom Stoof and others in Chapter 19, but some practical tips for setting up model systems would have been valuable in this context.

It was also disappointing not to see more attention given to serum-free culture of human cells. There are undoubtedly some limitations in serum-free culture, both from the point of view of the cell types that can be maintained, and from economic considerations; serum may be expensive, but is still, regrettably, cheaper and more readily available than serumfree recipes.
The Handbook is accompanied by a spiral-bound methods book, Keratinocyte Methods, which has a number of very useful protocols and would be indispensable if you wished to put some of the methods discussed in the Handbook into practice. This is a useful way to present this type of publication as the more expensive review component need not be taken into the laboratory while the smaller methods component, being spiral bound, lends itself to bench use, and would be cheaper to replace when the phenol red stains eventually obliterated the text! However, the relative proportions of the two do not seem quite in balance and I would have preferred to see a larger version of the Methods book with more consistent and detailed protocols, some of which are very brief, with more complete information on the concentration and preparation of reagents, more precise details, such as the absolute concentration of cells at subculture, and the sources of materials.

Like the Handbook, the Methods is divided into logical sections to guide you into the areas that you require. Taken together, these two books would be a valuable asset to the keratinocyte biologist. There is good coverage on the types of methods employed, details of which are available in the companion volume Keratinocyte Methods. The Keratinocyte Handbook is well illustrated and indexed, has a very elegant presentation and was a pleasure to review. It is, however, rather expensive at $£ 130$, even with the Methods included.

RI Freshney

CRC Dept of Medical Oncology Alexander Stone Building

Garscube Estate, Switchback Road Bearsden, Glasgow G61 1BD 